OPEN ACCESS

Edited by:

Aiping Lu,

Hong Kong Baptist University, China

Reviewed by:

Subhalakshmi Ghosh,

Jadavpur University, India

Cheng Xiao,

China-Japan Friendship Hospital,

China

*Correspondence:

Chi-Meng Tzeng

cmtzeng@xmu.edu.cn

Specialty section:

This article was submitted to

Ethnopharmacology,

a section of the journal

Frontiers in Pharmacology

Received: 20 December 2015

Accepted: 15 February 2016

Published: 01 March 2016

Citation:

Li Z, Lin H, Gu L, Gao J and Tzeng

C-M (2016) Herba Cistanche (Rou

Cong-Rong): One of the Best

Pharmaceutical Gifts of Traditional

Chinese Medicine.

Front. Pharmacol. 7:41

doi: 10.3389/fphar.2016.00041

\section{Herba Cistanche (Rou Cong-Rong): One of the Best Pharmaceutical Gifts of Traditional Chinese Medicine}

\author{
Zhiming $\mathrm{Li}^{1,2}$, Huinuan $\mathrm{Lin}^{1}$, Long Gu${ }^{1}$, Jingwen Gao ${ }^{1}$ and Chi-Meng Tzeng ${ }^{1,2,3 *}$ \\ ${ }^{1}$ Translational Medicine Research Center, School of Pharmaceutical Sciences, Xiamen University, Xiamen, China, ${ }^{2}$ Key \\ Laboratory for Cancer T-Cell Theranostics and Clinical Translation, Xiamen, China, ${ }^{3}$ INNOVA Clinics and TRANSLA Health \\ Group, Xiamen, China
}

Cistanche species, known as Rou Cong-Rong in Chinese, are an endangered wild species and are mainly distributed in the arid lands and warm deserts of northwestern China. Within Traditional Chinese Medicine (TCM), Herba Cistanche is applied as a tonic and/or in a formula for chronic renal disease, impotence, female infertility, morbid leucorrhea, profuse metrorrhagia, and senile constipation. The chemical constituents of Herba Cistanche mainly consist of volatile oils, nonvolatile phenylethanoid glycosides (PhGs), iridoids, lignans, alditols, oligosaccharides, and polysaccharides. There have been an increasing number of studies focusing on its bio-activities, including antioxidation, neuroprotection, and antiaging. The objective of this review is to introduce this herb to the world. Its taxonomy, distribution, and corresponding biological functions and molecular mechanisms are addressed in this review.

Keywords: Cistanche species, Herba Cistanche, phenylethanoid glycosides, improvement of brain function, aphrodisiac effect, immune-boosting effect

Cistanche Hoffmg. Et Link is a genus within the Orobanchaceae family and includes 22 species throughout the world. The cistanche species include the perennial parasite herbs, which commonly attach onto the roots of sand-fixing plants, such as Haloxylon ammodendron, H. persicum, Kalidium foliatum, and Tamarix plants (Li et al., 2013b). Generally, cistanche species distribute in arid lands and deserts in the northern hemisphere, such as the provinces of Xinjiang, Inner Mongolia, Gansu, Qinghai, and the Ningxia Autonomous Region in China in addition to similar regions of countries such as Iran, India, and Mongolia (Jiang and Tu, 2009). The growth and cultivation of cistanche species require severe environmental conditions: extreme arid climate, depauperate soils, large temperature difference, intensive sunshine, and less than $250 \mathrm{~mm}$ of annual precipitation (Qiao et al., 2007). Among the 22 species in the world, six are found in China according to the Taxonomical Index of Chinese Higher Plants (Plant Institute of Chinese Academy of Science, 1994); however, a follow-up study indicated that only 4 species and 1 variation of cistanche exist in China, including Cistanche deserticola Y. C. Ma, C. tubulosa (Schenk) R. Wight, C. salsa (C. A. Mey.) G. Beck, C. salsa var. albiflora P. F. Tu et Z. C. Lou and C. sinensis G. Beck (Jiang and $\mathrm{Tu}, 2009$ ).

The Herba Cistanche (Rou Cong-Rong in Chinese) was first recorded in Shen Nong's Chinese Materia Medica, where it was referred to as the dried succulent stems of the cistanche species (Karalliedde and Kappagoda, 2009). Among all the tonics in traditional Chinese medicine (TCM), Herba Cistanche is widely accepted as a superior one and has even been given the name "Ginseng of the deserts." In TCM, Herba Cistanche is frequently prescribed to treat chronic renal disease, 


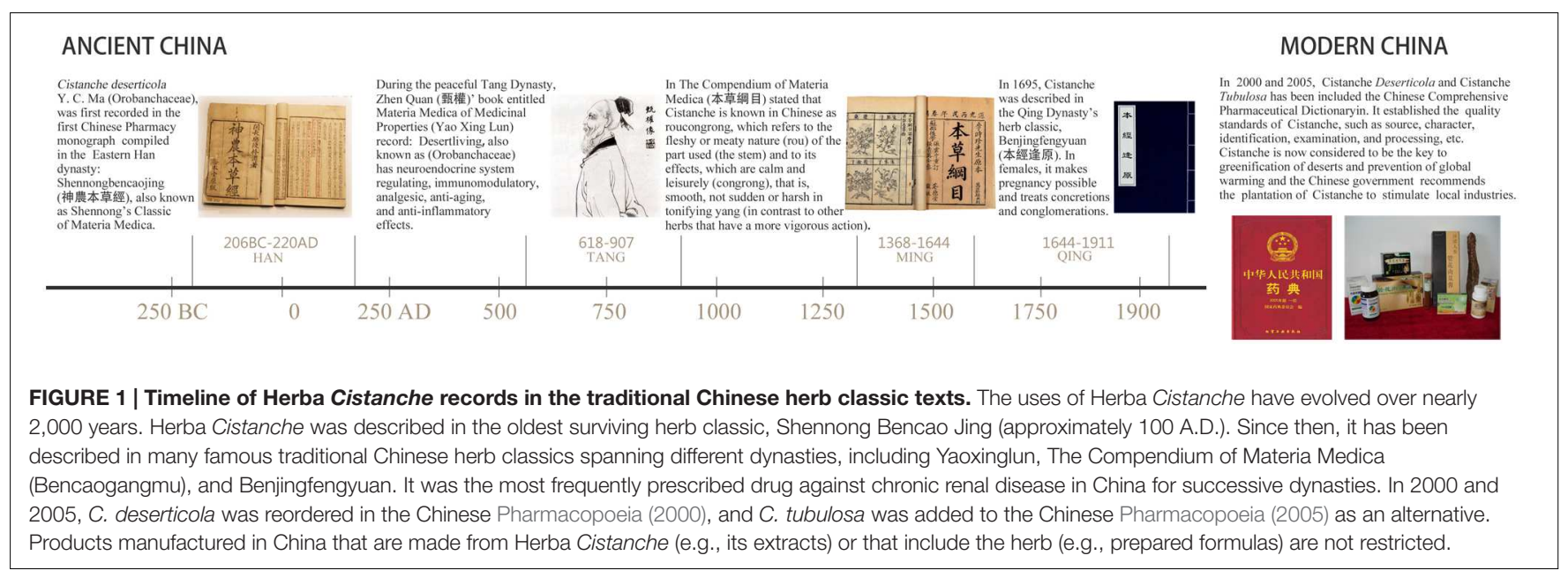

impotence, female infertility, morbid leucorrhea, profuse metrorrhagia, and senile constipation (Zhang et al., 2005). In 2000 and 2005, respectively, C. tubulosa, and C. deserticola were indexed in the Chinese Pharmacopeia (Pharmacopoeia, 2000) (Figure 1). C. tubulosa is offered as an alternative for C. deserticola because of its similar chemical constituents and pharmacological activities and its abundance (Pharmacopoeia, 2005). Other species of this genus, e.g., C. salsa and C. sinensis, are also used as alternatives in some areas. Cistanche is considered a new cultivated plant in several regions in northwestern China, where the rainfall is low and soil desertification is severe.

Since the 1980s, researchers have been interested in Herba Cistanche. A chemical analysis of Herba Cistanche revealed that essential oils, phenylethanoid glycosides (PhGs), iridoids, lignans, alditols, oligosaccharides, cistanosides, and polysaccharides were the main constituents (Jin and Zhang, 1994). Herba Cistanche extracts are pharmacologically active, with a range of functions that include improving chronic renal disease and senile constipation, increasing learning/memorizing ability, treating Alzheimer's disease (AD), and improving immunity (Snytnikova et al., 2012; Zhang et al., 2012; Guo et al., 2013; Li et al., 2013a; Nan et al., 2013). Most pharmaceutical companies are hoping to find the next 'miracle' drug, such as artemisinin (qinghaosu), an antimalarial drug that is extracted from the medicinal plant sweet wormwood and has saved millions of lives. The other classic example of a modern medicine that originated in TCM is arsenic trioxide, which was approved by the US Food and Drug Administration (FDA) to treat leukemia in 2000. The present overview focuses on the progress of the study of chemical constituents of Herba Cistanche and some of its relevant pharmacological activities.

\section{CHEMICAL CONSTITUENTS}

\section{Volatile Compounds}

In essential oil of the Herba Cistanche, alkanes, alcohols, aldehydes, and heterocyclics were detected, and palmitic acid, linoleic acid, 14-methylpentadecanoate, ethyl palmitate, and 2,5,6-trimethyloctane were identified. The total oils of C. tubulosa mainly consist of palmitic acid and linoleic acid, while the essential oil of C. salsa includes alkanes, alcohols, aldehydes, and some heterocyclic compounds (Jiang and $\mathrm{Tu}, 2009$ ). The volatile compounds of Herba Cistanche, like the essential oils, commonly can be extracted by steam distillation or lipophilic organic solvent. By extracting $C$. deserticola with petroleum ether and then analyzing the sample on a GC-MS, 25 volatile compounds were identified, and the 3 richest constituents in the petroleum ether extract are methyl 14-methylpentadecanoate (13.61\%), ethyl palmitate (12.39\%), and 2,5,6-trimethyloctane (7.60\%) (Jiang and Tu, 2009).

\section{Non-Volatile Compounds}

Among the non-volatile compounds of Herba Cistanche, more than 100 have currently been isolated and identified. These compounds are mostly PhGs, iridoids, lignans, alditols, oligosaccharides, and polysaccharides. As an important class of the compounds that constitute Herba Cistanche, PhGs have been well studied (Table 1). To date, $34 \mathrm{PhG}$ compounds have been successfully isolated from Herba Cistanche, including 22 disaccharide glycosides, 10 trisaccharide glycosides, and 2 monosaccharide glycosides. The empirical structural features of PhGs are as follows. (1) For disaccharide glycosides, the sugar moiety consists of glucose and rhamnose connected by a Glc $(3 \rightarrow 1)$ Rha linkage; the glucose commonly links directly to an aglycone, and a coumaroyl or caffeoyl is usually located at the C4 or C6 position. (2) For trisaccharide glycosides, there is another glucose or rhamnose at the C6 position of the inside glucose. Recently, Li et al. (2015) completed the first deep transcriptome sequencing of the fleshy stem of $C$. deserticola by RNA-seq and identified some key enzyme genes and pathways that are involved in the biosynthesis of lignin and PhGs, which provides valuable information for this medical plant.

For the other non-volatile compounds of Herba Cistanche, 3 iridoid aglycones and 14 iridoid glycosides have currently been isolated from cistanche species (Xie et al., 2006); 1 and 5 lignan 


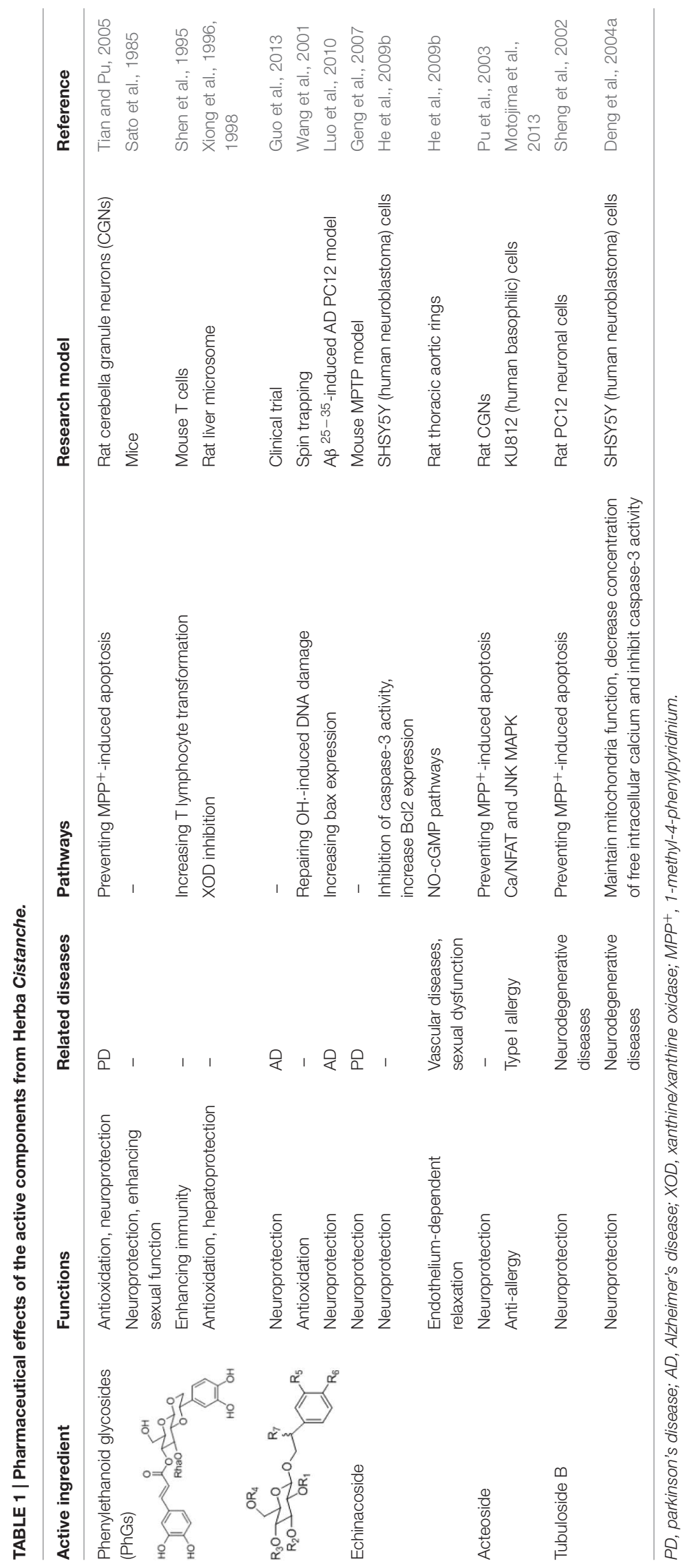


glycosides have been isolated from C. deserticola and C. tubulosa, respectively. Only 2 alkaloids, betaine and N,N-dimethyl glycine methyl ester, have been isolated from Herba Cistanche (Jiang and $\mathrm{Tu}, 2009$ ). Small amounts of other compounds, such as phenolic glycosides, sterols or their glycosides, fatty acids, amino acids, and trace elements, are also present in Herba Cistanche (Snytnikova et al., 2012).

\section{PHARMACOLOGICAL ACTIVITIES}

Based on the neuroprotection, immune-enhancement, and sexual health properties of Herba Cistanche, we had a discussion in the paper (Supplementary Figure S1).

\section{Improvement of Brain Function}

When considering learning and memory, three levels of mechanisms are involved: (1) the ability to acquire memory, i.e., learning ability; (2) the ability to store memory, i.e., consolidation; and (3) the ability to recall memorized information (Choi et al., 2011). The C. tubulosa extract was confirmed to significantly improve these mechanisms by preventing brain neuron apoptosis through the expression of apoptosis-related factors and neurotrophic factors in MES23.5 cells (Lin et al., 2013). C. tubulosa extract, containing rich echinacoside, and acteoside, can alleviate the cognitive dysfunction caused by $A \beta^{1-42}$ through blocking amyloid deposition, reversing cholinergic, and hippocampal dopaminergic neuronal function in $\mathrm{AD}$-like rat model (Wu et al., 2014). One of the PhGs, echinacoside, is typically known as the main phenolic component in the roots of Echinacea angustifolia, which is widely used in Europe and North America for its immunoregulation properties. A recent study determined that echinacoside can rescue human fibroblasts (SHSY5Y) from $\mathrm{TNF} \alpha$-induced apoptosis. The results indicated that echinacoside protects the damaged fibroblasts by regulating the reactive oxygen species level in fibroblasts and the activation of caspase-3 (Zhao et al., 2010). In vitro and in vivo experiments have confirmed that individual PhGs can inhibit the apoptosis of neuronal cells induced by various chemicals (Tian and $\mathrm{Pu}$, 2005; Geng et al., 2007). Therefore, the individual PhGs could be attractive candidates against some typical neurodegenerative disorders, such as dementia or Parkinson's disease (PD). Moreover, an open-label, non-placebo-controlled study on C. tubulosa glycoside capsules (Memoregain ${ }^{\circledR}$ ) demonstrates that the drug has a potential to be a possible treatment option for mild to moderate $\mathrm{AD}$, and all adverse reactions were mild. (Guo et al., 2013).

Similarly, the C. salsa extract can accelerate the proliferation of fibroblasts and promote the production of neurons by accelerating the growth of the neurites. It also has some properties as follows: prevent damage caused by cerebral ischemiareperfusion; protect against apoptosis of the CA1 region of hippocampus (Wang et al., 2004); and increase the amount of neurotransmitters, such as dopamine (DA), noradrenaline (NA), and serotonin (5-HT), in the rat brain (Chen et al.,
2007; Choi et al., 2011; Zhong et al., 2012). The acteosiderich fraction of $C$. salsa extract can inhibit reactive oxygen species, prevent DNA damage, enhance superoxide dismutase (SOD) activity and prevent lipid peroxidation (Lin et al., 2002; Deng et al., 2004b; He et al., 2009a). Because acteoside has an extremely strong antioxidative effect that is 15 times stronger than resveratrol and five times stronger than vitamin $\mathrm{C}$ (Chiou et al., 2004).

\section{Aphrodisiac Effect}

A recent study has demonstrated that an ethanol extract of C. tubulosa could increase the sex hormone levels by inducing testicular steroidogenic enzymes (e.g., CYP11A1, CYP17A1, CYP3A4) (Wang et al., 2015). Besides, the gene expression of $3 \beta$-hydroxysteroid dehydrogenase ( $3 \beta$-HSD), which is responsible for the synthesis of testosterone, $5 \alpha$-reductase- 2 and aldo-keto reductase (enzymes that are responsible for the synthesis of dihydrotestosterone), can be induced by the C. tubulosa extract, suggesting the positive effect of C. tubulosa extract on male hormone production (Shimoda et al., 2009). The acteoside, which was distilled from C. tubulosa, significantly shortened the latent period of penis erection $(p<0.01)$, increased the number of germ cells $(p<0.01)$, and improved pathological changes in the testes (Ma et al., 2009). Echinacoside, which is another newly identified $\mathrm{PhG}$ and is typically known as the main component of echinacea, is abundant in C. tubulosa and possesses vaso-relaxing activity (Yoshikawa et al., 2006). The aphrodisiac mechanisms of $C$. tubulosa might involve the NO-cGMP signal transduction pathway, with increasing cGMP levels in the corpus cavernosum smooth muscle ( $\mathrm{He}$ et al., 2009b). Pan and Min (2004) indicated that the combined use of Herba Cistanche extract could prevent adrenal cortical atrophy, which is typically caused by using corticosteroids only. Moreover, $C$. deserticola extract could reverse the reproductive toxicity in mice induced by hydroxyurea (Gu et al., 2013) and glycoside of Leigongteng (Radix et Rhizoma Tripterygii) (Li et al., 2014).

According to TCM, Yang-Qi Kidney-Yang Deficiency Syndrome (KDS-Yang) is caused by insufficient "Yang-Qi" in the kidney. Briefly, Yang-Qi is a TCM term and likely indicates mitochondrion-driven biological activities of the human body in the view of biomedical research (Leong et al., 2015). In H9c2 cardiomyocytes, Herba Cistanche was proven to enhance the mitochondrial respiration and glutathione antioxidant status (Wong and Ko, 2013). Yang-Qi deficiencies in TCM resemble those of chronic fatigue syndrome in Western medicine. KDS-Yang symptoms include soreness and weakness of the waist and knees, cold chills, deafness, and tinnitus. Modern studies showed that damage and functional disorders of the hypothalamic-pituitary-target gland axis, including the adrenal gland, thyroid, and gonad, are the main pathological mechanisms of KDS-Yang (Zhao et al., 2013). Gong et al. (2008) investigated the intervention effect of $C$. deserticola (decoction, $10 \mathrm{~g} / \mathrm{kg} \cdot \mathrm{d}$ ) in hydrocortisone-induced KDS-Yang model rats and found that C. deserticola extract could increase body weight, autonomic activity, and swimming time while decreasing post-exercise blood lactic acid (LAC) and blood urea nitrogen (BUN). As 
described in the Compendium of Materia Medica, Herba Cistanche is mild and would not be extremely abrupt or harsh when treating KDS-Yang. Interestingly, KDS-Yang also leads to low and disordered immune function, and thus, there is a close relationship between treating KDS-Yang and improving immune function (Yim and Ko, 2002).

\section{Immune-Boosting Effect}

Besides its traditional use, the daily consumption of Herba Cistanche is believed to the key of the people with longevity in some region of China and Japan where known for longevity and oasis. Studies shown that both C. salsa extract and C. tubulosa extract can activate lymphoid cells and increase the killed rate of cancer cells (Maruyama et al., 2009). C. deserticola extract can activate the phagocytic function of macrophages in mice and enhance body immunity (Li et al., 2009). Carbohydrates account for a high proportion of the dry mass of Herba Cistanche. The polysaccharides of C. deserticola are closely related to the immunity enhancing and anti-cancer functions (Xu et al., 2011). Galactitol is one of the monosaccharides in Herba Cistanche with laxative activity (Baishun et al., 2003). Individual compounds such as oligosaccharides present an excellent effect on the spleen activity of mice, increase the phagocytotic activity of macrophages and stimulate the proliferation of antibody-producing cells (Maruyama et al., 2008). Acteoside (10 or $50 \mathrm{mg} / \mathrm{kg}$ subcutaneously) significantly inhibits hepatic apoptosis, hepatitis and lethality in mice with hepatic apoptosis and liver failure were induced by D-galactosamine (DGalN) and lipopolysaccharide (LPS) (Xiong et al., 1999). Echinacosideenriched extract of $C$. tubulosa is effective in preventing dextran sulphate sodium (DSS)-induced colitis in mice (Jia et al., 2014). Recently, Zhang et al. (2014) discovered that C. deserticola extract could antagonize immune-related senescence and extend the lifespan in SAM-P8 mice. In Zhang's et al. (2014) study, dietary supplementation with C. deserticola extract can decrease the level of peripheral memory $\mathrm{T}$ cells and enhance levels of naive $\mathrm{T}$ cells. Additionally, Herba Cistanche aqueous extract was proven to prevent bone loss caused by ovarian hormone deficiency through regulating some bone metabolism related genes (e.g., Smad1, Smad5, TGF-b1, and TIEG1) (Liang et al., 2011, 2013), and amonoterpene from $C$. salsa has been identified as an anti-osteoporotic compound (Yamaguchi et al., 1999). Herba Cistanche helps people better understand the synergistic effect mechanisms of the effective components in TCM.

\section{REFERENCES}

Baishun, Z., Shuanghou, C., and Xuewen, Z. (2003). Study on the dosage-effect relationship of laxative action of galactitol from cistanche deserticola [J]. Chin. J. Inform. 12:15.

Chen, H., Jing, F. C., Li, C. L., Tu, P. F., Zheng, Q. S., and Wang, Z. H. (2007). Echinacoside prevents the striatal extracellular levels of monoamine neurotransmitters from diminution in 6-hydroxydopamine lesion rats. J. Ethnopharmacol. 114, 285-289. doi: 10.1016/j.jep.2007.07.035

Chiou, W. F., Lin, L. C., and Chen, C. F. (2004). Acteoside protects endothelial cells against free radical-induced oxidative stress. J. Pharm. Pharmacol. 56, 743-748. doi: 10.1211/0022357023501

\section{CONCLUSION}

Herba Cistanche has been commonly used traditionally for enhancing immunity, sexual health, antioxidation, and neuroprotection, and tonic. Various Herba Cistanche products and its derivative are widely used in modern China. The uses of this drug have evolved over nearly 2,000 years, and the demand for Herba Cistanche has grown rapidly in recent years. After a long-term use, those compounds with proven pharmacological activity, such as acteoside, and echinacoside, deserve more indepth study before they truly could improve patient quality of life. With further study, this important TCM is believed to have profound prospects.

\section{AUTHOR CONTRIBUTIONS}

HL drafted the table. LG and JG designed the figure. ZL and CT drafted and revised the manuscript.

\section{SUPPLEMENTARY MATERIAL}

The Supplementary Material for this article can be found online at: http://journal.frontiersin.org/article/10.3389/fphar. 2016.00041

FIGURE S1 | Main biological functions and mechanisms of the active components of Herba Cistanche. The active ingredients isolated from Herba Cistanche have three main biological functions: improvement of brain function, immune-boosting effect, and nourishing aphrodisiac effect. Herba Cistanche extracts are mixtures of multiple active compounds, and a typical traditional Chinese medicine (TCM) recognizes multiple biological targets. Phenylethanoid glycosides (PhGs) have neuroprotective properties in neurodegenerative disease based on their free radical scavenging ability, anti-apoptosis activity, anti-inflammation activity, the increasing amount of brain neurotransmitters and enhanced SOD activity. A modern use of Herba Cistanche in Chinese herbalism is to treat KDS-Yang, which contributes to fertility problems (including impotence and female infertility) because Herba Cistanche is different from other herbs that often have a more vigorous action. Herba Cistanche regulates the hypothalamic-pituitary-gonad (HPG) and hypothalamic-pituitary-adrenal (PHA) axes, which may induce a smooth and balanced sexual vitality effect. Moreover, phenylethanoid oligoglycosides and acylated oligosaccharides from Herba Cistanche have vasorelaxant activity via the NO-cGMP pathway. Based on clinical and experimental data, activation of the HPA axis increases plasma cytokines and acute phase protein concentrations. Thus, it can increase phytohemagglutinin-stimulated lymphocyte proliferation and enhance antibody production in response to T-dependent antigens in spleen cells.

Choi, J. G., Moon, M., Jeong, H. U., Kim, M. C., Kim, S. Y., and Oh, M. S. (2011) Cistanches Herba enhances learning and memory by inducing nerve growth factor. Behav. Brain Res. 216, 652-658. doi: 10.1016/j.bbr.2010.09.008

Deng, M., Zhao, J. Y., Ju, X. D., Tu, P. F., Jiang, Y., and Li, Z. B. (2004a). Protective effect of tubuloside B on TNF alpha-induced apoptosis in neuronal cells. Acta Pharmacol. Sin. 25, 1276-1284.

Deng, M., Zhao, J. Y., Tu, P. F., Jiang, Y., Li, Z. B., and Wang, Y. H. (2004b). Echinacoside rescues the SHSY5Y neuronal cells from TNF $\alpha$-induced apoptosis. Eur. J. Pharmacol. 505, 11-18. doi: 10.1016/j.ejphar.2004.09.059

Geng, X., Tian, X., Tu, P., and Pu, X. (2007). Neuroprotective effects of echinacoside in the mouse MPTP model of Parkinson's disease. Eur. J. Pharmacol. 564, 66-74. doi: 10.1016/j.ejphar.2007.01.084 
Gong, M. J., Liu, X. M., and Wang, L. W. (2008). Study on antifatigue effect of caulis cistanchis on the mice with yang Asthenia. Guid. J. 13, 8-10.

Gu, L., Xiong, W. T., Wang, C., Sun, H. X., Li, G. F., and Liu, X. (2013). Cistanche deserticola decoction alleviates the testicular toxicity induced by hydroxyurea in male mice. Asian J. Androl. 15, 838-840. doi: 10.1038/aja.2013.73

Guo, Q., Zhou, Y., Wang, C. J., Huang, Y. M., Lee, Y. T., Su, M. H., et al. (2013). An open-label, nonplacebo-controlled study on Cistanche tubulosa glycoside capsules (Memoregain(R)) for treating moderate Alzheimer's Disease. Am. J. Alzheimers Dis. Other Demen. 28, 363-370. doi: 10.1177/1533317513488907

He, W., Fang, T., and Tu, P. (2009a). Research progress on pharmacological activities of echinacoside. Zhongguo Zhong Yao Za Zhi 34, 476-479.

He, W. J., Fang, T. H., Ma, X., Zhang, K., Ma, Z. Z., and Tu, P. F. (2009b). Echinacoside elicits endothelium-dependent relaxation in rat aortic rings via an NO-cGMP pathway. Planta Med. 75, 1400-1404. doi: 10.1055/s-0029-1185745

Jia, Y., Guan, Q., Jiang, Y., Salh, B., Guo, Y., Tu, P., et al. (2014). Amelioration of dextran sulphate sodium-induced colitis in mice by echinacoside-enriched extract of Cistanche tubulosa. Phytother. Res. 28, 110-119. doi: 10.1002/ptr.4967

Jiang, Y., and Tu, P. F. (2009). Analysis of chemical constituents in Cistanche species. J. Chromatogr. A 1216, 1970-1979. doi: 10.1016/j.chroma.2008.07.031

Jin, X. L., and Zhang, Q. R. (1994). Recent progress in the study on chemical constituents of herba Cistanche. Zhongguo Zhong Yao Za Zhi 19, 695-697.

Karalliedde, L. D., and Kappagoda, C. T. (2009). The challenge of traditional Chinese medicines for allopathic practitioners. Am. J. Physiol. Heart Circ. Physiol. 297, H1967-H1969. doi: 10.1152/ajpheart.00944.2009

Leong, P. K., Wong, H. S., Chen, J., and Ko, K. M. (2015). Yang/Qi invigoration: an herbal therapy for chronic fatigue syndrome with yang deficiency? Evid. Based Comp. Alternat. Med. 2015:945901. doi: 10.1155/2015/945901

Li, F., Yang, X., Yang, Y., Guo, C., Zhang, C., Yang, Z., et al. (2013a). Antiosteoporotic activity of echinacoside in ovariectomized rats. Phytomedicine 20, 549-557. doi: 10.1016/j.phymed.2013.01.001

Li, J., Huang, D., and He, L. (2014). Effect of roucongrong (Herba Cistanches Deserticolae) on reproductive toxicity in mice induced by glycoside of Leigongteng (Radix et Rhizoma Tripterygii). J. Tradit. Chin. Med. 34, 324-328. doi: 10.1016/S0254-6272(14)60097-2

Li, X., Ma, Y. Q., Song, Y. X., Shui, J. F., and Li, X. W. (2009). Effect of different plant growth regulators on transplanting survival ratio of Haloxylon ammodendron seedling and parasitic ratio of Cistanche deserticola. Zhong Yao Cai 32, 16511654.

Li, X., Zhang, T. C., Qiao, Q., Ren, Z., Zhao, J., Yonezawa, T., et al. (2013b). Complete chloroplast genome sequence of holoparasite Cistanche deserticola (Orobanchaceae) reveals gene loss and horizontal gene transfer from its host Haloxylon ammodendron (Chenopodiaceae). PLoS ONE 8:e58747. doi: 10.1371/journal.pone.0058747

Li, Y., Wang, X., Chen, T., Yao, F., Li, C., Tang, Q., et al. (2015). RNA-Seq based de novo transcriptome assembly and gene discovery of cistanche deserticola fleshy stem. PLoS ONE 10:e0125722. doi: 10.1371/journal.pone.0125722

Liang, H., Yu, F., Tong, Z., and Huang, Z. (2011). Effect of Cistanches Herba aqueous extract on bone loss in ovariectomized rat. Int. J. Mol. Sci. 12, 50605069. doi: 10.3390/ijms12085060

Liang, H. D., Yu, F., Tong, Z. H., Zhang, H. Q., and Liang, W. (2013). Cistanches Herba aqueous extract affecting serum BGP and TRAP and bone marrow Smad1 mRNA, Smad5 mRNA, TGF-betal mRNA and TIEG1 mRNA expression levels in osteoporosis disease. Mol. Biol. Rep. 40, 757-763. doi: 10.1007/s11033-012-2065-2

Lin, L.-W., Hsieh, M.-T., Tsai, F.-H., Wang, W.-H., and Wu, C.-R. (2002). Antinociceptive and anti-inflammatory activity caused by Cistanche deserticola in rodents. J. Ethnopharmacol. 83, 177-182. doi: 10.1016/S0378-8741(02)00147-2

Lin, S., Ye, S., Huang, J., Tian, Y., Xu, Y., Wu, M., et al. (2013). How do Chinese medicines that tonify the kidney inhibit dopaminergic neuron apoptosis? Neural Regen. Res. 8, 2820-2826.

Luo, L., Aerziguli, T., and Wang, X. W. (2010). Protective effects of glycosides of cistanche on apoptosis of PC12 cells induced by aggregated $\beta$-amyloid protein 25-35. Chin J. New Drugs Clin. Rem. 29, 115-118.

Ma, J. J., Zhao, F., and Sun, Y. (2009). The effects of acteoside on nourishing kidney and strengthening Yang in Yang deficient mice. J. Yangzhou Univ. 30, 22-25.

Maruyama, S., Akasaka, T., Yamada, K., and Tachibana, H. (2009). Bioactivity of high-molecular-weight constituents of Cistanche tubulosa. Pharmacology 1, 913-921.
Maruyama, S., Hashizume, S., Tanji, T., Yamada, K., and Tachibana, H. (2008). Cistanche salsa extract enhanced antibody production in human lymph node lymphocytes. Pharmacology 2, 341-348.

Motojima, H., Villareal, M. O., Iijima, R., Han, J., and Isoda, H. (2013). Acteoside inhibits type Iota allergy through the down-regulation of Ca/NFAT and JNK MAPK signaling pathways in basophilic cells. J. Nat. Med. 67, 790-798. doi: 10.1007/s11418-013-0753-4

Nan, Z. D., Zeng, K. W., Shi, S. P., Zhao, M. B., Jiang, Y., and Tu, P. F. (2013). Phenylethanoid glycosides with anti-inflammatory activities from the stems of Cistanche deserticola cultured in Tarim desert. Fitoterapia 89, 167-174. doi: 10.1016/j.fitote.2013.05.008

Pan, Y. R., and Min, F. Y. (2004). The influence of the adrenal gland of kidney Yang deficiency animal model after treating Cistanche alcohol extract. J. Pract. Tradit. Chin. Med. 20, 357-357.

Pharmacopoeia, N. C. O. C. (2000). Pharmacopoeia of the People's Republic of China. Beijing: The Chemical Industry Publishing House.

Pharmacopoeia, N. C. O. C. (2005). Pharmacopoeia of the People's Republic of China. Beijing: The Chemical Industry Publishing House.

Plant Institute of Chinese Academy of Science (1994). Taxonomical Index of Chinese Higher Plants. Beijing: Publisher of Science, 401.

Pu, X. P., Song, Z. H., Li, Y. Y., Tu, P. F., and Li, H. N. (2003). Acteoside from Cistanche salsa inhibits apoptosis by 1-methyl-4-phenylpyridinium ion in cerebellar granule neurons. Planta Med. 69, 65-66. doi: 10.1055/s-200337029

Qiao, X. Y., Wang, H. L., and Guo, Y. H. (2007). Study on conditions of seed germination of Cistanche. Zhongguo Zhong Yao Za Zhi 32, 1848-1850.

Sato, T., Kozima, S., Kobayashi, K., and Kobayashi, H. (1985). Pharmacological studies on Cistanchis Herba. I. Effects of the constituents of cistanchis herba on sex and learning behavior in chronic stress mice (1). Yakugaku Zasshi 105, 1131-1144.

Shen, H. M., Wang, X. W., Gu, D. T., Li, L. L., and Wang, X. F. (1995). Morphological changes of peripheral blood corpuscles of radiated rats feeded with Cistanche. J. Xinjiang Med. Coll. 18, 83-89.

Sheng, G. Q., Pu, X. P., Lei, L., Tu, P. F., and Li, C. L. (2002). Tubuloside B from Cistanche salsa rescues the PC12 neuronal cells from 1-methyl-4phenylpyridinium ion-induced apoptosis and oxidative stress. Planta Med. 68, 966-970. doi: 10.1055/s-2002-35667

Shimoda, H., Tanaka, J., Takahara, Y., Takemoto, K., Shan, S. J., and Su, M. H. (2009). The hypocholesterolemic effects of Cistanche tubulosa extract, a Chinese traditional crude medicine, in mice. Am. J. Chin. Med. 37, 1125-1138. doi: 10.1142/S0192415X09007545

Snytnikova, O. A., Tsentalovich, Y. P., Stefanova, N. A., Fursova, A., Kaptein, R., Sagdeev, R. Z., et al. (2012). The therapeutic effect of mitochondria-targeted antioxidant SkQ1 and Cistanche deserticola is associated with increased levels of tryptophan and kynurenine in the rat lens. Dokl. Biochem. Biophys. 447, 300-303. doi: 10.1134/S1607672912060087

Tian, X.-F., and Pu, X.-P. (2005). Phenylethanoid glycosides from Cistanches salsa inhibit apoptosis induced by 1-methyl-4-phenylpyridinium ion in neurons. J. Ethnopharmacol. 97, 59-63. doi: 10.1016/j.jep.2004.10.014

Wang, T., Chen, C., Yang, M., Deng, B., Kirby, G. M., and Zhang, X. (2015). Cistanche tubulosa ethanol extract mediates rat sex hormone levels by induction of testicular steroidgenic enzymes. Pharm. Biol. 54, 481-487. doi: 10.3109/13880209.2015.1050114

Wang, X., Meng, X., and Jiang, X. (2004). Protective effects of glycosides of cistanche on cerebral ischemia-reperfusion damage of brain tissue in CA_1 region of hippocumpus in awake mice. Stroke Nerv. Dis. 10, 325-328.

Wang, X. W., Jiang, X. Y., Wu, L.-Y., Wang, X. F., and Abdulrahim-Yusup. (2001). Scavenging effects of glycosides of Cistanche on free radicals and its protection against $\mathrm{OH}$-induced DNA damage in vitro. J. Clin. Pharmacol. 36:4.

Wong, H. S., and Ko, K. M. (2013). Herba Cistanches stimulates cellular glutathione redox cycling by reactive oxygen species generated from mitochondrial respiration in H9c2 cardiomyocytes. Pharm. Biol. 51, 64-73. doi: 10.3109/13880209.2012.710242

Wu, C. R., Lin, H. C., and Su, M. H. (2014). Reversal by aqueous extracts of Cistanche tubulosa from behavioral deficits in Alzheimer's disease-like rat model: relevance for amyloid deposition and central neurotransmitter function. BMC Comp. Altern. Med. 14:202. doi: 10.1186/1472-6882-14-202 
Xie, H., Morikawa, T., Matsuda, H., Nakamura, S., Muraoka, O., and Yoshikawa, M. (2006). Monoterpene constituents from Cistanche tubulosachemical structures of kankanosides A-E and kankanol. Chem. Pharm. Bull. (Tokyo) 54, 669-675. doi: 10.1248/cpb.54.669

Xiong, Q., Hase, K., Tezuka, Y., Namba, T., and Kadota, S. (1999). Acteoside inhibits apoptosis in D-galactosamine and lipopolysaccharideinduced liver injury. Life Sci. 65, 421-430. doi: 10.1016/S0024-3205(99) 00263-5

Xiong, Q. B., Hase, K., Tezuka, Y., Tani, T., Namba, T., and Kadota, S. (1998). Hepatoprotective activity of phenylethanoids from Cistanche deserticola. Planta Med. 64, 120-125. doi: 10.1055/s-2006-957387

Xiong, Q. B., Kadota, S., Tani, T., and Namba, T. (1996). Antioxidative effects of phenylethanoids from Cistanche deserticola. Biol. Pharm. Bull. 19, 1580-1585. doi: 10.1248/bpb.19.1580

Xu, H., Wei, X. D., Ou, Q., Li, Y., Xu, X. X., Wang, J. T., et al. (2011). Desertliving Cistanche anti-aging action: a comparative study. Heilongjiang Med. Pharm. 34, $1-2$.

Yamaguchi, K., Shinohara, C., Kojima, S., Sodeoka, M., and Tsuji, T. (1999). (2E,6R)-8-hydroxy-2,6-dimethyl-2-octenoic acid, a novel anti-osteoporotic monoterpene, isolated from Cistanche salsa. Biosci. Biotechnol. Biochem. 63, 731-735. doi: 10.1271/bbb.63.731

Yim, T., and Ko, K. (2002). Antioxidant and immunomodulatory activities of Chinese tonifying herbs. Pharm. Biol. 40, 329-335. doi: 10.1076/phbi.40.5.329.8457

Yoshikawa, M., Matsuda, H., Morikawa, T., Xie, H., Nakamura, S., and Muraoka, O. (2006). Phenylethanoid oligoglycosides and acylated oligosugars with vasorelaxant activity from Cistanche tubulosa. Bioorg. Med. Chem. 14, 7468-7475. doi: 10.1016/j.bmc.2006.07.018

Zhang, C. Z., Wang, S. X., Zhang, Y., Chen, J. P., and Liang, X. M. (2005). In vitro estrogenic activities of Chinese medicinal plants traditionally used for the management of menopausal symptoms. J. Ethnopharmacol. 98, 295-300. doi: 10.1016/j.jep.2005.01.033
Zhang, K., Ma, X., He, W., Li, H., Han, S., Jiang, Y., et al. (2014). Extracts of Cistanche deserticola can antagonize immunosenescence and extend life span in senescence-accelerated mouse prone 8 (SAM-P8) Mice. Evid. Based Comp. Altern. Med. 2014:601383. doi: 10.1155/2014/ 601383

Zhang, X., Wu, F., Lin, R., Zhang, X., Liu, N., Li, J., et al. (2012). Changes of lung, spleen and kidney aquaporin-1 in rats with Kidney Yang Deficiency: the "water metabolism theory" in traditional Chinese medicine. Nan Fang Yi Ke Da Xue Xue Bao 32, 1507-1510.

Zhao, L., Wu, H., Qiu, M., Sun, W., Wei, R., Zheng, X., et al. (2013). Metabolic signatures of kidney yang deficiency syndrome and protective effects of two herbal extracts in rats using GC/TOF MS. Evid. Based Comp. Alternat. Med. 2013:540957. doi: 10.1155/2013/540957

Zhao, Q., Gao, J., Li, W., and Cai, D. (2010). Neurotrophic and neurorescue effects of Echinacoside in the subacute MPTP mouse model of Parkinson's disease. Brain Res. 1346, 224-236. doi: 10.1016/j.brainres.2010. 05.018

Zhong, M., Chen, H., Jiang, Y., Tu, P. F., Liu, C. L., Zhang, W. X., et al. (2012). Effects of echinacoside on striatal extracellular levels of amino acid neurotransmitter in cerebral ischemia rats. Chin. Pharmacol. Bull. 28, $361-365$.

Conflict of Interest Statement: The authors declare that the research was conducted in the absence of any commercial or financial relationships that could be construed as a potential conflict of interest.

Copyright (c) 2016 Li, Lin, Gu, Gao and Tzeng. This is an open-access article distributed under the terms of the Creative Commons Attribution License (CC BY). The use, distribution or reproduction in other forums is permitted, provided the original author(s) or licensor are credited and that the original publication in this journal is cited, in accordance with accepted academic practice. No use, distribution or reproduction is permitted which does not comply with these terms. 